BMJ Open

Diabetes

Research

\& Care

\section{Additive effects of green tea and coffee on all-cause mortality in patients with type 2 diabetes mellitus: the Fukuoka Diabetes Registry}

To cite: Komorita Y, Iwase M, Fujii $\mathrm{H}$, et al. Additive effects of green tea and coffee on all-cause mortality in patients with type 2 diabetes mellitus: the Fukuoka Diabetes Registry. BMJ Open Diab Res Care 2020;8:e001252. doi:10.1136/ bmjdrc-2020-001252

Received 5 February 2020 Revised 3 July 2020 Accepted 13 August 2020
Check for updates

(C) Author(s) (or their employer(s)) 2020. Re-use permitted under CC BY-NC. No commercial re-use. See rights and permissions. Published by BMJ.

${ }^{1}$ Department of Medicine and Clinical Science, Graduate School of Medical Sciences, Kyushu University, Fukuoka, Japan

${ }^{2}$ Division of Internal Medicine, Fukuoka Dental College,

Fukuoka, Japan

${ }^{3}$ Diabetes Center, Hakujyuji Hospital, Fukuoka, Japan

Correspondence to Dr Masanori Iwase; iwase@intmed2.med.kyushuu.ac.jp

\section{ABSTRACT}

Introduction The impact of consuming green tea or coffee on mortality in patients with diabetes is controversial. We prospectively investigated the impact of each beverage and their combination on mortality among Japanese patients with type 2 diabetes.

Research design and methods In all, 4923 patients (2790 men, 2133 women) with type 2 diabetes (mean age, 66 years) were followed prospectively (median, 5.3 years; follow-up rate, $99.5 \%$ ). We evaluated the amount of green tea and coffee consumed using self-administered questionnaires.

Results During the follow-up period, 309 participants died. The consumption of green tea, coffee, and a combination of the beverages was associated with reduced all-cause mortality. Multivariable-adjusted hazard ratios $(95 \%$ Cls) for green tea were as follows: none 1.0 (referent); 0.85 (0.60-1.22) for $\leq 1$ cup/day; 0.73 (0.51-1.03) for $2-3$ cups/day; $0.60(0.42-0.85)$ for $\geq 4$ cups/day; and $P$ for trend, 0.002 . For coffee, they were: none 1.0 (referent); $0.88(0.66-1.18)$ for $<1$ cup/day; 0.81 (0.58-1.13) for 1 cup/day; 0.59 (0.42-0.82) for $\geq 2$ cups/ day; $P$ for trend, 0.002 . With the combination they were 1.0 (referent) for no consumption of green tea and coffee; 0.49 (0.24-0.99) for $2-3$ cups/day of green tea with $\geq 2$ cups/ day of coffee; $0.42(0.20-0.88)$ for $\geq 4$ cups/day of green tea with $1 \mathrm{cup} /$ day of coffee; and $0.37(0.18-0.77)$ for $\geq 4$ cups/day of green tea with $\geq 2$ cups/day of coffee. Conclusions Higher consumption of green tea and coffee was associated with reduced all-cause mortality: their combined effect appeared to be additive in patients with type 2 diabetes.

\section{INTRODUCTION}

Worldwide, the number of patients with diabetes is increasing in both developed and developing countries. ${ }^{1}$ Diabetic patients are at increased risk for classical micro- and macrovascular diseases as well as nonclassical diseases, such as dementia, malignancy, and fragility fracture, which affect both quality of life and life expectancy. ${ }^{2}$ Recent advances in therapeutics (such as sodium-glucose

\section{Significance of this study}

What is already known about this subject?

- Higher green tea or coffee consumption has been reported to confer health benefits, and reduce all-cause mortality in the general population, respectively.

- No studies have investigated the association between green tea consumption and mortality in diabetic patients, and few studies have examined the association between coffee consumption and mortality in diabetic patients, and the results are controversial.

What are the new findings?

- Higher consumption of green tea and coffee was associated with reduced all-cause mortality.

- The combined effect of green tea and coffee on mortality appeared to be additive in patients with type 2 diabetes.

How might these results change the focus of research or clinical practice?

- This study suggests that consuming green tea and coffee may have beneficial effects on the longevity of Japanese people with type 2 diabetes.

cotransporter 2 inhibitors, glucagon-like peptide-1 receptor agonists, and bariatric surgery) have been remarkable, however, lifestyle improvements are still regarded as the basic therapy. ${ }^{3}$ Lifestyle management comprises physical activity, smoking cessation, and nutrition therapy (including both food and beverages). ${ }^{3}$ However, epidemiological research on nonalcoholic beverages, such as green tea and coffee, has been scarce for diabetic patients.

Asian, especially East Asian, populations have a traditional habit of drinking green tea. Green tea derives from fresh leaves of Camellia sinensis, and it contains various chemicals, such as phenolic compounds, theanine, 
and caffeine. ${ }^{4}$ Through antioxidant, anti-inflammatory, or anti-bacterial properties, ${ }^{45}$ higher green tea consumption has been reported to confer health benefits, including preventing such chronic diseases as diabetes mellitus, ${ }^{46}$ and reduce mortality. ${ }^{78}$ Hitherto, however, no studies have investigated the association between green tea consumption and mortality in diabetic patients.

Coffee is one of the most frequently consumed beverages worldwide. It contains numerous bioactive chemicals, including phenolic compounds and caffeine, that have been reported to impact health through various mechanisms, such as antioxidant, anti-inflammatory, and anti-mutagenic effects: ${ }^{9}{ }^{10}$ the result is a reduced risk of developing type 2 diabetes, dyslipidemia, or malignancy. ${ }^{10}$ In contrast to these health-promoting effects, coffee also has harmful effects, such as increasing blood pressure $^{10}$ and the risk of myocardial infarction. ${ }^{11}$ In the general population, there is growing evidence that coffee consumption is associated with reduced risk of all-cause and cause-specific mortality. ${ }^{12-15}$ However, few epidemiological studies have examined this association in diabetic patients, and the results are controversial. ${ }^{16-19}$ This discrepancy may be due to the presence of diabetic complications, including cardiovascular disease (CVD), which could be affected by caffeine consumption. ${ }^{11}$ Further, most studies have been conducted only with developed country populations, which have higher risks of CVD than Asian ones. ${ }^{20}$

Recently, green tea has attracted considerable attention as a healthy beverage in developed countries. ${ }^{21}$ Japan has the culinary culture of both its own traditional food with green tea and developed country food with coffee: this provides a unique opportunity to investigate the combined effect of green tea and coffee consumption on mortality. Accordingly, in the present study, we prospectively investigated the impact of green tea, coffee consumption, and their combination on all-cause and cause-specific mortality in Japanese patients with type 2 diabetes.

\section{RESEARCH DESIGN AND METHODS \\ Study participants}

The Fukuoka Diabetes Registry is a multicenter prospective study (UMIN Clinical Trial Registry 000002627) designed to investigate the effect of modern treatments and lifestyles on the prognoses of patients with diabetes mellitus. The registry includes patients who regularly attended teaching hospitals certified by the Japan Diabetes Society or certified diabetes clinics in Fukuoka Prefecture, Japan. ${ }^{22}$ In all, 5131 patients with diabetes mellitus aged 20 years or older were registered during regular visits between April 2008 and October 2010. The registry exclusion criteria were as follows: patients with drug-induced diabetes or those undergoing corticosteroid treatment; patients undergoing renal replacement therapy; patients with serious diseases other than diabetes, such as advanced malignancies and decompensated liver cirrhosis; and patients unable to visit diabetologists regularly. After excluding 208 participants with type 1 diabetes, we enrolled the remaining 4923 participants in this study. The study conformed with the principles of the Declaration of Helsinki and was approved by the Kyushu University Institutional Review Board (approval number 290). All participants provided their written informed consent to take part.

\section{Clinical evaluation at registration as baseline}

Information regarding diabetes duration from its diagnosis, smoking habits, alcohol intake, leisure-time physical activity (LTPA), sleep duration, depressive symptoms, and history of coronary heart disease (CHD), stroke, and cancer was obtained using a self-administered questionnaire. Smoking habits and alcohol intake were classified as either current or not. LTPA was estimated as metabolic equivalent-hours per week. ${ }^{23}$ We assessed the presence of depressive symptoms using the Center for Epidemiologic Studies Depression Scale: $:^{24}$ participants who scored 16 or more among 60 points were defined as having depressive symptoms.

Body weight and height were measured, and body mass index (BMI) was calculated. Blood pressure was measured in a sitting position: hypertension was defined as blood pressure $\geq 140 / 90 \mathrm{mmHg}$ or current use of antihypertensive agents. Medical charts were reviewed for all medications, including insulin, oral hypoglycemic agent, antihypertensive drugs, antiplatelet drugs, and statins.

The dietary survey, including green tea and coffee consumption, was conducted using a self-administered brief diet history questionnaire (Gender Medical Research Inc., Tokyo) regarding the frequency of $58 \mathrm{food}$ items and supplements. The validity of many nutrients has previously been studied in an adult Japanese population. ${ }^{25}$ Green tea and coffee consumption was self-reported and assessed by answering the question "How often do you drink each beverage?" Participants were asked to select one among eight categories: none; $<1 \mathrm{cup} /$ week; $1 \mathrm{cup} /$ week; 2-3 cups/week; 4-6 cups/week; 1 cup/day; 2-3 cups/day; and $\geq 4$ cups/day. The amounts of green tea and coffee consumed differed: green tea, none $12 \%, \leq 1$ cup/day $23 \%, \geq 2$ cups/day $64 \%$; and coffee, none $20 \%$, $\leq 1 \mathrm{cup} /$ day $46 \%, \geq 2 \mathrm{cups} /$ day $34 \%$. Accordingly, we categorized the participants into the following four groups by beverage: green tea, none, $\leq 1 \mathrm{cup} /$ day, $2-3 \mathrm{cups} /$ day, $\geq 4$ cups/day; and coffee, none, $<1 \mathrm{cup} /$ day, $1 \mathrm{cup} /$ day, $\geq 2$ cups/day. There were no questions about the consumption of decaffeinated or caffeinated drinks because decaffeinated beverages are uncommon in Japan.

\section{Laboratory measurements}

Blood samples were collected by venipuncture, and spot urine samples were obtained. Hemoglobin $\mathrm{A}_{1 \mathrm{c}}\left(\mathrm{HbA}_{1 \mathrm{c}}\right)$ was determined by high-performance liquid chromatography (Tosoh Corp., Tokyo,). Serum low-density lipoprotein (LDL) cholesterol, high-density lipoprotein (HDL) cholesterol, creatinine, and urinary creatinine 
concentrations were measured using enzymatic methods. We measured urinary albumin using an immunonephelometry method (Medical and Biological Laboratories Co., Ltd., Nagoya). We calculated estimated glomerular filtration rates (eGFR) based on serum creatinine using the equation proposed by the Japanese Society of Nephrology. ${ }^{26}$ Urinary albumin-creatinine ratio (UACR) levels were calculated by dividing the urinary albumin values by the urinary creatinine concentrations.

\section{Mortality follow-up}

The primary outcome in this study was all-cause mortality. Participants received an annual follow-up during their survival through interviews, medical records, letters, telephone calls, and municipal registration of residence. During the follow-up period (median, 5.3 years), 27 participants were lost to follow-up (follow-up rate, $99.5 \%$ ). The underlying cause of death was determined based on medical records or death certificates: it was coded according to the International Classification of Diseases, 10th revision.

\section{Statistical analysis}

The age- and sex-adjusted mortality rate was calculated using the person-years method: we calculated mortality in each 10-year age-group by sex and then weighted it based on the distribution of all participants. The Cox proportional-hazards models were used to estimate the HRs and 95\% CIs for mortality. In the multivariableadjusted model, we selected the following covariates, known to be a potential risk or protective factors for mortality: age, sex, BMI, diabetes duration, current smoking habit, current alcohol intake, LTPA, sleep duration, $\mathrm{HbA}_{1 c}$, UACR, systolic blood pressure, LDL cholesterol, history of CVD, and cancer. We conducted further adjustment for the coffee-drinking habit to analyze the association between green tea and mortality, and vice versa. We confirmed the proportional hazard assumption graphically and using the Schoenfeld residual test: the P-value was 0.57 for the green tea category, 0.44 for the coffee category, and 0.15 for the green tea and coffee category. All statistical analyses were performed using Statistical Analysis Software (SAS) version 9.4 (SAS institute Inc., Cary, NC, USA). We considered values of $\mathrm{P}<0.05$ statistically significant in all analyses.

\section{RESULTS}

Table 1 summarizes the baseline clinical characteristics of the four groups divided according to the amount of green tea or coffee consumed. With the green tea groups, mean age, duration of diabetes, sleep duration, HDL cholesterol, systolic blood pressure, and frequency of cancer history increased in participants with higher green tea consumption. By contrast, the proportion of males, current smokers and current drinkers, eGFR, and diastolic blood pressure decreased in participants with higher green tea consumption. With the coffee groups, mean age, sleep duration, UACR, systolic blood pressure, frequency of taking antihypertensive or antiplatelet drugs, history of CVD, and cancer decreased in participants with higher coffee consumption. Conversely, the proportion of males, current smokers and current drinkers, mean LTPA, HbA ${ }_{1 c}$ eGFR, LDL cholesterol, HDL cholesterol, and diastolic blood pressure increased in participants with higher coffee consumption.

During the follow-up period (median, 5.3 years; follow-up rate, 99.5\%), 309 participants (218 men, 91 women) died. The main causes of death were cancer $(\mathrm{n}=114)$ and CVD $(\mathrm{n}=76)$. Table 2 presents the number of all-cause and cause-specific deaths, crude incidence, multivariable-adjusted HRs, and 95\% CIs according to the green tea and coffee consumption groups. In the green tea groups, all-cause mortality significantly decreased according to the amount of green tea consumed ( $\mathrm{P}$ for trend, 0.001). The multivariable-adjusted HR was significantly lower in participants who consumed $\geq 4$ cups/day of green tea compared with those who did not drink green tea (HR, $0.60 ; 95 \%$ CI, 0.42 to 0.85 ). Further, the HR remained significant after additional adjustments for coffee consumption. Cause-specific mortality for cancer was not associated with the amount of green tea consumed: the association of the amount of green tea consumed with CVD was marginally insignificant ( $\mathrm{P}$ for trend, 0.08). In the coffee groups, all-cause mortality was also significantly reduced according to the amount of coffee consumed ( $\mathrm{P}$ for trend, 0.001). The multivariableadjusted HR was significantly lower in participants who consumed $\geq 2$ cups/day of coffee than in those who did not consume coffee (HR, 0.59; 95\% CI, 0.42 to 0.82). That association remained significant after further adjustments for green tea consumption. Cause-specific mortality for cancer was not associated with the amount of coffee consumed: the association of the amount of coffee consumption with CVD was marginally insignificant ( $\mathrm{P}$ for trend, 0.06).

Figure 1 shows the multivariable-adjusted HRs of allcause mortality according to combined green tea and coffee consumption. When the mortality in participants drinking neither green tea nor coffee was set as the referent, multivariable-adjusted HRs were significantly reduced as follows: in those consuming 2-3 cups of green tea with $\geq 2$ cups/day of coffee (HR, $0.49 ; 95 \%$ CI, 0.24 to 0.99 ); consuming $\geq 4$ cups/day of green tea with 1 cup/day of coffee (HR, 0.42; $95 \%$ CI, 0.20 to 0.88 ); and drinking $\geq 4$ cups of green tea with $\geq 2$ cups/day of coffee (HR, $0.37 ; 95 \%$ CI, 0.18 to 0.77 ) (table 3 ).

\section{CONCLUSIONS}

In this prospective study, we found that higher green tea and coffee consumption was significantly associated with decreased all-cause mortality in patients with type 2 diabetes. This association remained significant after adjusting for potential confounders: the impact of each beverage on mortality was independent. Similar trends were evident in analyzes of CVD-specific mortality. 
Table 1 Baseline clinical characteristics according to amount of green tea or coffee consumed among Japanese participants with type 2 diabetes

\begin{tabular}{|c|c|c|c|c|c|c|c|c|}
\hline & \multicolumn{4}{|c|}{ Green tea consumption } & \multicolumn{4}{|c|}{ Coffee consumption } \\
\hline & None & $\leq 1 \mathrm{cup} / \mathrm{d}$ & $2-3$ cups/d & $\geq 4$ cups $/ d$ & None & $<1$ cup /d & $1 \mathrm{cup} / \mathrm{d}$ & $\geq 2$ cups $/ d$ \\
\hline$N$ & 607 & 1143 & 1389 & 1784 & 994 & 1306 & 963 & 1660 \\
\hline Age (years) & $64.6 \pm 10.2$ & $62.5 \pm 10.8$ & $65.7 \pm 9.8$ & $67.3 \pm 9.7$ & $69.0 \pm 10.0$ & $66.4 \pm 10.2$ & $65.6 \pm 10.1$ & $62.3 \pm 9.5$ \\
\hline Male sex (\%) & 60.3 & 64.3 & 60.5 & 47.6 & 50.6 & 54.7 & 53.3 & 63.9 \\
\hline $\begin{array}{l}\text { Duration of diabetes } \\
\text { (years) }\end{array}$ & $14.9 \pm 10.3$ & $14.8 \pm 10.4$ & $15.9 \pm 10.6$ & $16.0 \pm 10.6$ & $16.3 \pm 10.9$ & $15.5 \pm 10.6$ & $15.5 \pm 10.3$ & $15.2 \pm 10.4$ \\
\hline $\mathrm{BMI}\left(\mathrm{kg} / \mathrm{m}^{2}\right)$ & $23.9 \pm 3.4$ & $24.1 \pm 3.8$ & $23.5 \pm 3.7$ & $23.7 \pm 3.9$ & $23.7 \pm 3.7$ & $23.9 \pm 3.8$ & $23.9 \pm 4.0$ & $23.6 \pm 3.6$ \\
\hline Current smoker (\%) & 24.9 & 20.7 & 18.85 & 15.1 & 12.6 & 12.1 & 15.1 & 29.2 \\
\hline Current alcohol intake (\%) & 40.5 & 45.7 & 42.6 & 31.8 & 30.5 & 38.8 & 40.9 & 43.5 \\
\hline $\begin{array}{l}\text { Depressive symptoms } \\
(\%)\end{array}$ & 10.2 & 9.6 & 7.5 & 8.7 & 9.7 & 9.3 & 7.5 & 8.6 \\
\hline LTPA (MET h/week) & $12.3 \pm 16.1$ & $11.2 \pm 14.7$ & $11.5 \pm 14.0$ & $12.0 \pm 15.3$ & $11.3 \pm 14.8$ & $11.3 \pm 14.9$ & $12.5 \pm 15.2$ & $11.8 \pm 14.8$ \\
\hline Sleep duration (h/day) & $6.8 \pm 1.4$ & $6.7 \pm 1.3$ & $6.9 \pm 1.3$ & $6.9 \pm 1.5$ & $7.0 \pm 1.6$ & $6.9 \pm 1.4$ & $6.9 \pm 1.3$ & $6.7 \pm 1.3$ \\
\hline $\mathrm{HbA}_{1 \mathrm{c}}(\%)$ & $7.4 \pm 1.0$ & $7.4 \pm 1.0$ & $7.4 \pm 1.0$ & $7.5 \pm 1.1$ & $7.4 \pm 1.1$ & $7.4 \pm 1.0$ & $7.4 \pm 1.0$ & $7.5 \pm 1.1$ \\
\hline $\mathrm{HbA}_{1 \mathrm{c}}(\mathrm{mmol} / \mathrm{mol})$ & $57.3 \pm 10.8$ & $57.4 \pm 11.2$ & $57.4 \pm 10.7$ & $58.4 \pm 12.2$ & $57.4 \pm 11.8$ & $57.1 \pm 11.2$ & $57.8 \pm 11.0$ & $58.5 \pm 11.6$ \\
\hline eGFR $\left(\mathrm{ml} / \mathrm{min} / 1.73 \mathrm{~m}^{2}\right)$ & $73.3 \pm 23.7$ & $78.6 \pm 21.8$ & $74.2 \pm 21.4$ & $73.5 \pm 20.9$ & $69.7 \pm 23.1$ & $73.5 \pm 21.0$ & $75.5 \pm 22.2$ & $78.6 \pm 20.4$ \\
\hline $\mathrm{UACR}(\mathrm{mg} / \mathrm{g} \cdot \mathrm{Cr})$ & $246 \pm 780$ & $175 \pm 690$ & $178 \pm 583$ & $195 \pm 620$ & $251 \pm 771$ & $209 \pm 666$ & $189 \pm 695$ & $145 \pm 509$ \\
\hline LDL cholesterol (mmol/l) & $2.9 \pm 0.7$ & $2.8 \pm 0.7$ & $2.9 \pm 0.7$ & $2.9 \pm 0.7$ & $2.8 \pm 0.7$ & $2.8 \pm 0.7$ & $2.8 \pm 0.7$ & $2.9 \pm 0.7$ \\
\hline HDL cholesterol (mmol/l) & $1.4 \pm 0.4$ & $1.5 \pm 0.4$ & $1.5 \pm 0.4$ & $1.5 \pm 0.4$ & $1.4 \pm 0.4$ & $1.4 \pm 0.4$ & $1.5 \pm 0.4$ & $1.5 \pm 0.4$ \\
\hline $\begin{array}{l}\text { Systolic blood pressure } \\
(\mathrm{mmHg})\end{array}$ & $130 \pm 18$ & $130 \pm 17$ & $130 \pm 17$ & $132 \pm 17$ & $131 \pm 18$ & $132 \pm 17$ & $131 \pm 17$ & $129 \pm 16$ \\
\hline $\begin{array}{l}\text { Diastolic blood pressure } \\
(\mathrm{mmHg})\end{array}$ & $75 \pm 11$ & $75 \pm 11$ & $75 \pm 11$ & $74 \pm 11$ & $73 \pm 11$ & $74 \pm 11$ & $75 \pm 11$ & $75 \pm 10$ \\
\hline History of CVD (\%) & 24.2 & 22.1 & 20.7 & 21.4 & 25.7 & 22.8 & 20.9 & 18.9 \\
\hline History of cancer (\%) & 7.1 & 8.5 & 9.4 & 10.7 & 11.1 & 9.4 & 10.4 & 7.7 \\
\hline Insulin therapy (\%) & 32.8 & 27.7 & 27.0 & 29.6 & 30.4 & 24.9 & 28.7 & 31.1 \\
\hline $\begin{array}{l}\text { Oral hypoglycemic agent } \\
(\%)\end{array}$ & 59.6 & 64.2 & 65.5 & 62.3 & 61.2 & 64.9 & 64.1 & 62.9 \\
\hline $\begin{array}{l}\text { Antihypertensive drugs } \\
(\%)\end{array}$ & 55.7 & 51.6 & 54.6 & 54.4 & 61.9 & 57.4 & 56.5 & 45.1 \\
\hline Antiplatelet drugs (\%) & 28.2 & 22.9 & 24.1 & 25.8 & 29.4 & 26.9 & 25.2 & 20.6 \\
\hline Statin (\%) & 43.0 & 44.4 & 41.0 & 44.7 & 43.9 & 40.8 & 48.4 & 42.3 \\
\hline $\begin{array}{l}\text { Coffee drinker } \\
(>0 \text { cup/d, \%) }\end{array}$ & 75.1 & 87.0 & 81.5 & 75.5 & - & - & - & - \\
\hline $\begin{array}{l}\text { Green tea drinker } \\
\text { (>0 cup/d, \%) }\end{array}$ & - & - & - & - & 84.8 & 89.6 & 88.5 & 87.4 \\
\hline
\end{tabular}

Values are expressed as means \pm SD or percentages.

$\mathrm{BMI}$, body mass index; Cr, creatinine; CVD, cardiovascular disease; eGFR, estimated glomerular filtration rates; $\mathrm{HbA}_{1 \mathrm{c}}$, hemoglobin $A_{1 c}$; HDL, high-density lipoprotein; LDL, low-density lipoprotein; LTPA, leisure-time physical activity; MET, metabolic equivalent; UACR, urinary albumin creatinine ratio.

Further, the combination of higher green tea and coffee consumption significantly reduced all-cause mortality risk by $63 \%$.

Hitherto, little research has investigated the association between green tea consumption and mortality in diabetic patients. Some epidemiological studies from Japan and China have reported the association between green tea consumption and mortality in the general population, including diabetic and non-diabetic individuals. The
Ohsaki Study was the first large-scale investigation on this subject: among 40530 Japanese men and women, it found that those who consumed $\geq 5$ cups/day of green tea had a $15 \%$ lower risk of all-cause mortality than those drinking $<1 \mathrm{cup} /$ day. ${ }^{6} \mathrm{~A}$ meta-analysis of five cohort studies of the general population (including diabetic and nondiabetic individuals) demonstrated that the HRs $(95 \%$ CIs) of the highest vs the lowest green tea consumption were $0.80(0.68-0.93)$ for all-cause mortality and 0.67 


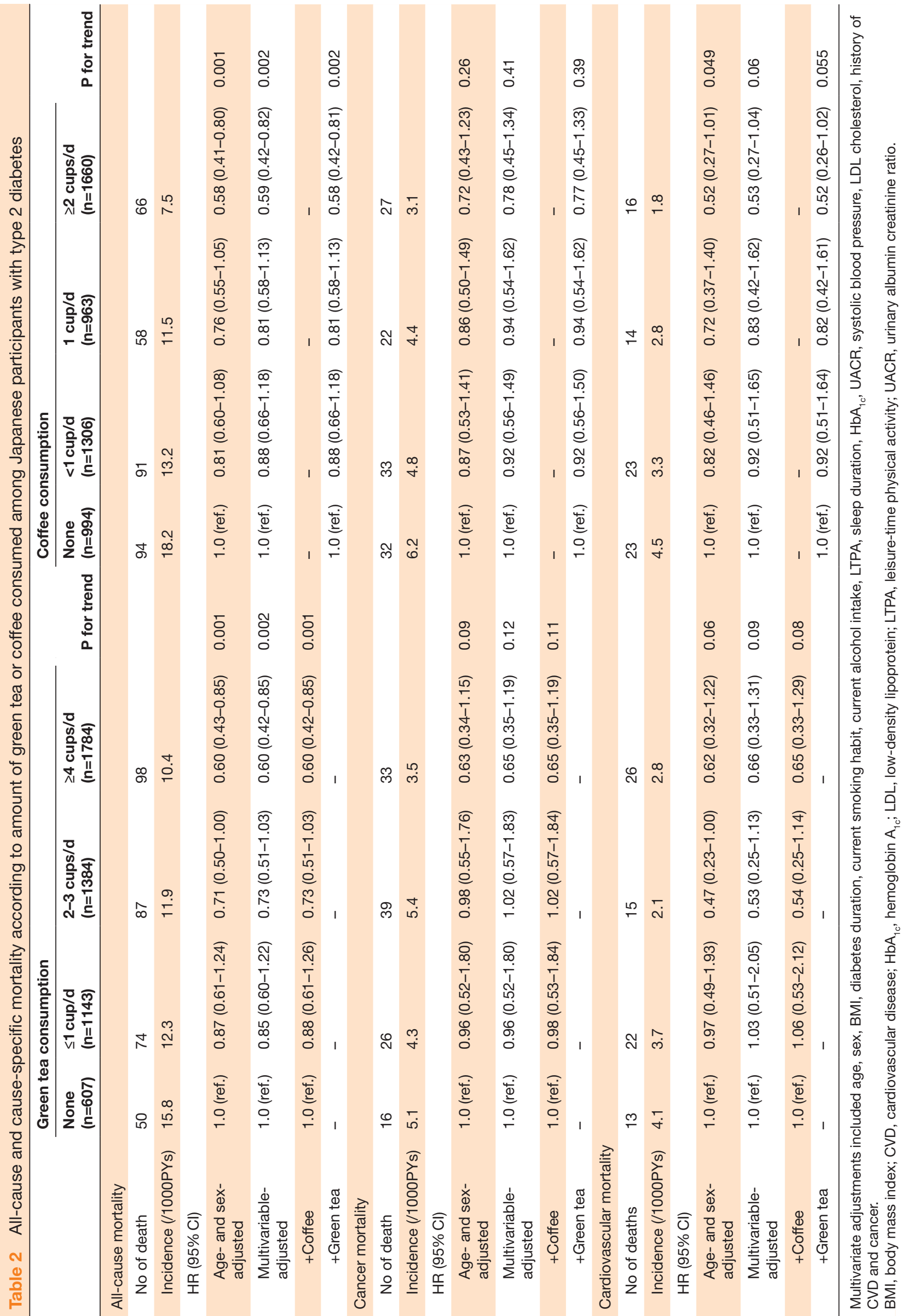




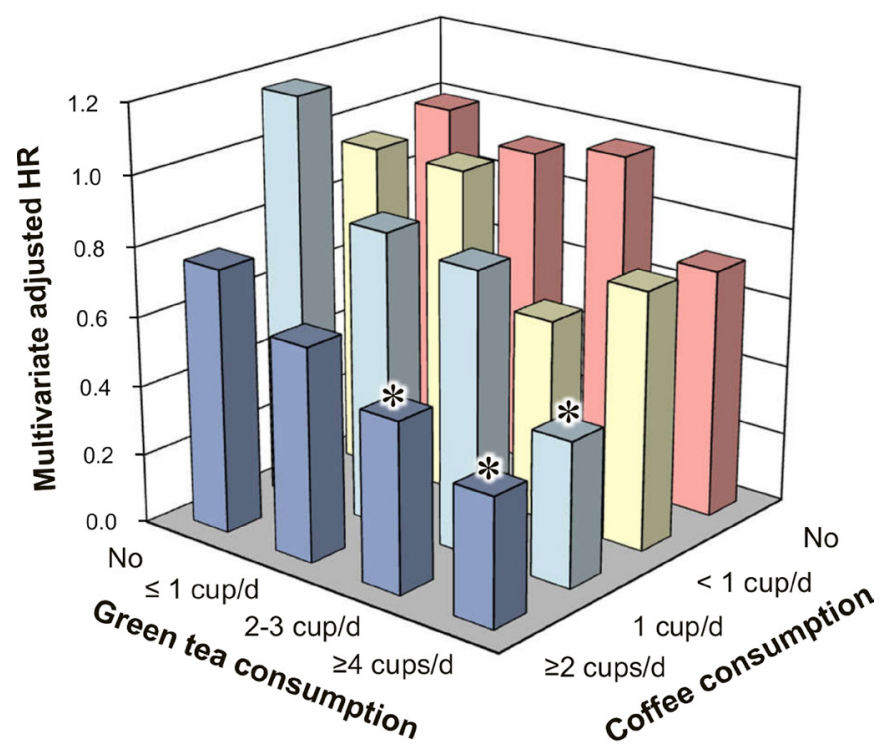

Figure 1 Combined effects of green tea and coffee consumption on all-cause mortality in patients with type 2 diabetes. The estimates are adjusted for age, sex, BMI, diabetes duration, current smoking habit, current alcohol intake, LTPA, sleep duration, $\mathrm{HbA}_{10}$, UACR, systolic blood pressure, LDL cholesterol, history of CVD and cancer. ${ }^{*} \mathrm{P}<0.05$ vs the reference group (NO consumption of green tea and coffee). BMI, body mass index; CVD, cardiovascular disease; $\mathrm{HbA}_{1 c}$, hemoglobin $\mathrm{A}_{1 c}$; $\mathrm{LDL}$, low-density lipoprotein; LTPA, leisure-time physical activity; UACR, urinary albumin creatinine ratio.

(0.46-0.96) for CVD mortality. ${ }^{7}$ The results of the present study are consistent with those of previous studies of the general population (including diabetic and non-diabetic individuals): however, we found the impact of green tea consumption to be even greater (HR for the highest vs lowest consumption, 0.60). To our knowledge, this is the first study to identify an association between higher green tea consumption and decreased all-cause mortality in type 2 diabetes patients.

The association between coffee consumption and mortality has been controversial in diabetic patients, although most studies have described beneficial effects in general populations (including diabetic and non-diabetic individuals). This association with diabetic patients was initially investigated with a Finnish diabetic population (3837 individuals) in 2006: a 30\% significant reduction in all-cause mortality was observed in those who consumed $\geq 7$ cups/day of coffee compared with those drinking 0-2 cups/day. ${ }^{16}$ However, the Health Professionals Follow-up Study of 3497 male diabetic patients found no association between coffee consumption and all-cause or CVD mortality ${ }^{17}$ The National Health and Nutrition Examination Survey recently found that $\geq 200 \mathrm{mg}$ /day of caffeine from coffee reduced all-cause mortality among 1974 women with diabetes, but not among men. ${ }^{19}$ Some largescale studies of general populations have attempted to identify associations in subgroup analyses of diabetic patients. The National Institutes of Health-American Association of Retired Persons Diet and Health Study (total $\mathrm{n}=246433$ ) found that consuming $\geq 4 \mathrm{cups} /$ day of coffee was associated with a $19 \%$ significant reduction of all-cause mortality in men and $14 \%$ insignificant reduction in women with self-reported diabetes. ${ }^{12}$ Similarly, in a US prospective cohort with 90317 participants, drinking $\geq 4$ cups/day of coffee was significantly associated with reduced all-cause mortality in diabetic patients. ${ }^{13}$

Table 3 Multivariate-adjusted HR and 95\% Cls of green tea and coffee consumption for all-cause mortality in patients with type 2 diabetes

\begin{tabular}{|c|c|c|c|c|}
\hline \multirow[b]{2}{*}{ Green tea consumption } & \multicolumn{4}{|c|}{ Coffee consumption } \\
\hline & No & $<1 \mathrm{cup} / \mathrm{d}$ & $1 \mathrm{cup} / \mathrm{d}$ & $\geq 2$ cups $/ \mathrm{d}$ \\
\hline \multicolumn{5}{|l|}{ No } \\
\hline Event no. /at risk & $14 / 151$ & $13 / 136$ & $11 / 111$ & $12 / 209$ \\
\hline $\mathrm{HR}(95 \% \mathrm{Cl})$ & 1.0 (ref.) & $0.95(0.45-2.04)$ & $1.17(0.53-2.59)$ & $0.76(0.35-1.66)$ \\
\hline \multicolumn{5}{|l|}{$\leq 1 \mathrm{cup} / \mathrm{d}$} \\
\hline Event no. /at risk & $17 / 149$ & $22 / 305$ & $15 / 230$ & $20 / 459$ \\
\hline $\mathrm{HR}(95 \% \mathrm{Cl})$ & $0.93(0.45-1.92)$ & $0.95(0.48-1.86)$ & $0.85(0.41-1.77)$ & $0.62(0.31-1.23)$ \\
\hline \multicolumn{5}{|l|}{$2-3$ cups/d } \\
\hline Event no. /at risk & $30 / 257$ & $20 / 361$ & $18 / 271$ & $19 / 500$ \\
\hline $\mathrm{HR}(95 \% \mathrm{Cl})$ & $0.98(0.52-1.86)$ & $0.59(0.30-1.16)$ & $0.81(0.40-1.64)$ & $0.49(0.24-0.99)$ \\
\hline \multicolumn{5}{|l|}{$\geq 4$ cups/d } \\
\hline Event no. /at risk & $33 / 437$ & $36 / 504$ & $14 / 351$ & $15 / 492$ \\
\hline HR (95\% Cl) & $0.72(0.38-1.35)$ & $0.74(0.40-1.38)$ & $0.42(0.20-0.88)$ & $0.37(0.18-0.77)$ \\
\hline
\end{tabular}

Multivariate adjustments include age, sex, BMI, diabetes duration, current smoking habit, current alcohol intake, LTPA, sleep duration, HbA ${ }_{1 \mathrm{c}}$, UACR, systolic blood pressure, LDL cholesterol, history of CVD and cancer.

$\mathrm{BMI}$, body mass index; CVD, cardiovascular disease; $\mathrm{HbA}_{1 \mathrm{c}}$, hemoglobin $\mathrm{A}_{1 \mathrm{c}}$; LDL, low-density lipoprotein; LTPA, leisure-time physical activity; UACR, urinary albumin creatinine ratio. 
Conversely, in a Dutch population, the Alpha Omega Study involving 4837 patients with prior myocardial infarction determined that consuming more than four cups/day of coffee increased mortality by $24 \%$ in diabetic patients, whereas it decreased mortality by $27 \%$ in those without diabetes. ${ }^{14}$ In the Nurses' Health Study (7170 female patients with diabetes), habitual coffee consumption was not found to be associated with increased risk of mortality. ${ }^{18}$ The UK Biobank (total $\mathrm{n}=498134$ participants) failed to identify a significant association between coffee consumption and mortality in diabetic patients. ${ }^{15}$ Most of the above studies were conducted in developed country populations, which have a higher risk of CVD than those in Asia. ${ }^{20}$ To our knowledge, the present investigation is the first prospective study to demonstrate a significant association between coffee consumption and reduced mortality in an Asian population with type 2 diabetes.

The mechanisms underlying reduced mortality with green tea and coffee consumption are not fully understood. Green tea contains a number of beneficial substances, including phenolic compounds, theanine, and caffeine. ${ }^{48}$ Epigallocatechin gallate is the most prevalent phenolic compound: it has been shown to have antioxidant, anti-inflammatory, and anti-mutagenic properties. ${ }^{27}$ Coffee also contains numerous bioactive components, including phenolic compounds and caffeine, which have been suggested as contributing to the associated favorable effects. ${ }^{9}$ Phenolic compounds, such as chlorogenic acid, have been reported to have a health impact through various mechanisms, such as antioxidant and anti-inflammatory actions. ${ }^{9} 28$

Caffeine is one of the components in green tea and coffee that could contribute to their favorable effects. The beneficial effects of caffeine on insulin sensitivity and glucose-induced insulin secretion have been suggested ${ }^{29}$ through several mechanisms, including weight loss and thermogenesis. ${ }^{30}{ }^{31}$ However, previous studies have found that consumption of both caffeinated and decaffeinated coffee was associated with reduced mortality. ${ }^{12}$ That could mean that caffeine does not play a beneficial role in the association between coffee consumption and risk of mortality. Caffeine has also been reported to stimulate sympathetic activity and increase serum catecholamine levels, ${ }^{32}$ leading to increased blood pressure or CVD risk. ${ }^{1133}$ The impact of coffee consumption on mortality was greater in the present study (HR 0.58 ) than that previously reported in developed country diabetic patients: HR 0.81 in men, ${ }^{12}$ HR $0.76,{ }^{13}$ HR $0.70,{ }^{16}$ and HR 0.53 in women. ${ }^{19}$ The difference may be explained by coffee having harmful effects among patients at higher CVD risk: that is based on findings that higher coffee consumption increased mortality among diabetic patients with previous myocardial infarction. ${ }^{14}$ Japanese type 2 diabetes patients are generally at lower CVD risk than developed ones: ${ }^{20}$ thus, caffeine may exert less detrimental effects on the vasculature, resulting in enhanced beneficial effects of coffee.
To date, no study has investigated the combined effect of green tea and coffee consumption on all-cause mortality. The present study determined that combined higher green tea and coffee consumption markedly reduced mortality. The Japan Public Health CenterBased Study found that the combination of $\geq 2$ cups/ day of green tea and $\geq 1 \mathrm{cup} /$ day of coffee significantly reduced the risk for cerebral infarction and intracerebral hemorrhage.$^{34}$ However, that effect did not apply to CHD: the study found no impact on CHD mortality. We found that the relative risk reduction of mortality (HR, $0.37)$ was greater than when consuming only green tea (HR, 0.72) or only coffee (HR, 0.76 ; figure 1 , table 3 ), thus, the protective mechanisms of the two beverages may differ. In addition to CVD and cancer, coffee reportedly confers health benefits with liver disease and dementia, ${ }^{10}$ however, reduced mortality associated with green tea is mostly explained with respect to CVD or cancer. ${ }^{78}$ Green tea and coffee consumption may be associated with different disease incidence or severity.

One strength of the present study is the high follow-up rate $(99.5 \%)$, which enabled us to accurately investigate the association among green tea and coffee consumption and mortality. Further, this cohort study included potential confounders, such as sleep duration, diabetic complications, lifestyle, physical activity, laboratory data, and medications.

Some limitations of this study deserve mention. First, green tea and coffee consumption was assessed using single, self-reported data, which raises the problem of potential misclassification. However, the green tea and coffee consumption evaluated using our questionnaire showed high correlation with that found with 16 nonconsecutive dietary records in a Japanese population. ${ }^{25}$ Second, we did not obtain information about some other confounding factors, such as educational and socioeconomic levels. Higher educational or income levels may be associated with greater coffee consumption: ${ }^{35}$ they may also be related to lower mortality risk. ${ }^{36}$ Those factors may be considered by adjusting for physical activities or smoking status, which could be associated with higher educational or income levels. ${ }^{36}$ However, a universal health insurance system has been established in Japan, thus, medical care is supposedly received regardless of educational or socioeconomic states. Third, the CVD medications differed among the coffee groups (table 1). Those medications were not considered in the analyses, however, history of CVD was included as a confounding factor. Fourth, we did not assess whether coffee was caffeinated or decaffeinated. However, decaffeinated coffee is uncommon in Japan. Previous studies have found a similar association between caffeinated or decaffeinated coffee and mortality. ${ }^{12}{ }^{13}$ Fifth, owing to the observational nature of the present study, we cannot conclude that consuming green tea or coffee reduces mortality. But long-term interventional studies of this kind may be difficult to implement: green tea and coffee are typically consumed as part of daily life. Sixth, the 
number of cause-specific deaths was relatively small. That could lead to low statistical power in detecting significant associations. Seventh, there may have been multiple testing problems in our statistical analyses. Multiple testing corrections were not conducted in the present study, however, the results of table 2 are evidently consistent with those of previous studies. ${ }^{153738}$ Finally, this study was conducted only among a Japanese population. The ingredients of Japanese green tea could differ from those in green tea in other countries. ${ }^{39}$ Accordingly, the findings of this study may not apply to other populations.

In conclusion, this prospective cohort study demonstrated that greater consumption of green tea and coffee was significantly associated with reduced all-cause mortality: the effects may be additive. Our results suggest that consuming green tea and coffee may have beneficial effects on the longevity of Japanese people with type 2 diabetes.

Acknowledgements The authors thank Drs. Dongchon Kang, Shuzo Kumagai, Shinako Ogata-Kaizu, Yoichiro Hirakawa, Akiko Sumi, Ai Murao-Kimura, Ayaka Oshiro (Kyushu University), Satoshi Sasaki (The University of Tokyo), Nobuhiro Sasaki (Fukuoka Red Cross Hospital), Kiyohide Nunoi, Yuichi Sato, Yuji Uchizono, Kaori Itoh (St. Mary's Hospital), Hirofumi Imoto (Steel Memorial Yawata Hospital), Daisuke Gotoh, Mina Matsuo (Kyushu Central Hospital), Sakae Nohara, Ayako Tsutsumi (Fukuoka Higashi Medical Center), Yasuhiro Idewaki (Hakujyuji Hospital), Masae Minami (Clinic Minami Masae), Miya Wada (Wada Miya Naika Clinic), Yoshifumi Yokomizo (Yokomizo Naika Clinic), Masanori Kikuchi, Yohei Kikuchi (Kikuchi Naika Clinic), Riku Nomiyama (Suzuki Naika Clinic), Shin Nakamura (Nakamura Naika Clinic), Kenji Tashiro (Oshima Eye Hospital), Mototaka Yoshinari (Yoshinari Naika Clinic), Kojiro Ichikawa (Fukutsu Naika Clinic), Yutaka Kiyohara, and Teruo Omae (Hisayama Research Institute for Lifestyle Diseases). The authors also thank clinical research coordinators Chiho Ohba (Hisayama Research Institute for Lifestyle Diseases), Yoko Nishioka (Kyushu University) as well as those in the administration office. Also, Tomoko Matake (Hisayama Research Institute for Lifestyle Diseases) and Junko Ishimatsu (Kyushu University). In addition, we thank Edanz Group (https://en-author-services.edanzgroup.com/) for editing a draft of this manuscript.

Contributors YK and MI were responsible for the study concept and design. YK, $\mathrm{Ml}$, and $\mathrm{HF}$ conducted the analysis. TO, HI, TJ-K, MY, YO, TH, UN, and TK interpreted the data and contributed to the discussion. YK and MI drafted the manuscript. All authors participated in critically revising the manuscript and approved the final version. Ml is the guarantor of this work and, as such, had full access to all the data in the study and takes responsibility for the integrity of the data and the accuracy of the data analysis.

Funding This work was supported in part by The Japan Society for the Promotion of Science KAKENHI from the Ministry of Education, Culture, Sports, Science and Technology of Japan (grant numbers 20K19663 to YK, 23249037 and 23659353 to MI, 16 K00861 to HF, and $19 K 24229$ to T0), the Junior Scientist Development Grant supported by the Japan Diabetes Society (to YK and T0), the Lilly Research Grant Program for Bone \& Mineral Research (to YK), a grant from the Japan Diabetes Foundation (to T0), and the Japan Heart Foundation and Astellas/Pfizer Grant for Research on Atherosclerosis Update (to T0).

Competing interests None declared.

Patient consent for publication Not required.

Ethics approval The Kyushu University Institutional Review Board (approval number 290). All participants provided their written informed consent to take part.

Provenance and peer review Not commissioned; externally peer reviewed.

Data availability statement The datasets generated during and/or analyzed during the current study are available from the corresponding author on reasonable request.

Open access This is an open access article distributed in accordance with the Creative Commons Attribution Non Commercial (CC BY-NC 4.0) license, which permits others to distribute, remix, adapt, build upon this work non-commercially, and license their derivative works on different terms, provided the original work is properly cited, appropriate credit is given, any changes made indicated, and the use is non-commercial. See: http://creativecommons.org/licenses/by-nc/4.0/.

ORCID iD

Masanori Iwase http://orcid.org/0000-0002-7510-4176

\section{REFERENCES}

1 Guariguata L, Whiting DR, Hambleton I, et al. Global estimates of diabetes prevalence for 2013 and projections for 2035. Diabetes Res Clin Pract 2014;103:137-49.

2 Kirkman MS, Briscoe VJ, Clark N, et al. Diabetes in older adults. Diabetes Care 2012;35:2650-64.

3 American Diabetes Association. 5. Lifestyle Management: Standards of Medical Care in Diabetes-2019. Diabetes Care 2019;42:S46-60.

4 Schneider C, Segre T. Green tea: potential health benefits. Am Fam Physician 2009;79:591-4.

5 Boehm K, Borrelli F, Ernst E, et al. Green tea (Camellia sinensis) for the prevention of cancer. Cochrane Database Syst Rev 2009:Cd005004.

6 Ninomiya T, Kanzaki N, Hirakawa Y, et al. Serum ethylamine levels as an indicator of -theanine consumption and the risk of type 2 diabetes in a general Japanese population: the Hisayama study. Diabetes Care 2019;42:1234-40.

7 Kuriyama S, Shimazu T, Ohmori K, et al. Green tea consumption and mortality due to cardiovascular disease, cancer, and all causes in Japan: the Ohsaki study. JAMA 2006;296:1255-65.

8 Tang J, Zheng J-S, Fang L, et al. Tea consumption and mortality of all cancers, CVD and all causes: a meta-analysis of eighteen prospective cohort studies. Br J Nutr 2015;114:673-83.

9 Martini D, Del Bo' C, Tassotti M, et al. Coffee consumption and oxidative stress: a review of human intervention studies. Molecules 2016;21:979.

10 Poole R, Kennedy OJ, Roderick P, et al. Coffee consumption and health: umbrella review of meta-analyses of multiple health outcomes. BMJ 2017;359:j5024.

11 Cornelis MC, El-Sohemy A, Kabagambe EK, et al. Coffee, CYP1A2 genotype, and risk of myocardial infarction. JAMA 2006;295:1135-41.

12 Freedman ND, Park Y, Abnet CC, et al. Association of coffee drinking with total and cause-specific mortality. N Engl J Med 2012;366:1891-904.

13 Loftfield E, Freedman ND, Graubard BI, et al. Association of coffee consumption with overall and cause-specific mortality in a large US prospective cohort study. Am J Epidemiol 2015;182:1010-22.

14 van Dongen LH, Mölenberg FJ, Soedamah-Muthu SS, et al. Coffee consumption after myocardial infarction and risk of cardiovascular mortality: a prospective analysis in the alpha omega cohort. $\mathrm{Am} \mathrm{J}$ Clin Nutr 2017;106:1113-20.

15 Loftfield E, Cornelis MC, Caporaso N, et al. Association of coffee drinking with mortality by genetic variation in caffeine metabolism: findings from the UK Biobank. JAMA Intern Med 2018;178:1086-97.

16 Bidel S, Hu G, Qiao Q, et al. Coffee consumption and risk of total and cardiovascular mortality among patients with type 2 diabetes. Diabetologia 2006;49:2618-26.

17 Zhang W, Lopez-Garcia E, Li TY, et al. Coffee consumption and risk of cardiovascular diseases and all-cause mortality among men with type 2 diabetes. Diabetes Care 2009;32:1043-5.

18 Zhang WL, Lopez-Garcia E, Li TY, et al. Coffee consumption and risk of cardiovascular events and all-cause mortality among women with type 2 diabetes. Diabetologia 2009;52:810-7.

19 Neves JS, Leitão L, Magriço R, et al. Caffeine consumption and mortality in diabetes: an analysis of NHANES 1999-2010. Front Endocrinol 2018;9:547.

20 Yokoyama $\mathrm{H}$, Matsushima M, Kawai K, et al. Low incidence of cardiovascular events in Japanese patients with type 2 diabetes in primary care settings: a prospective cohort study (JDDM 20). Diabet Med 2011;28:1221-8.

21 Statista: types of tea consumed at home in the U.S, 2017. Available: https://wwwstatistacom/statistics/679641/types-of-tea-consumedat-home-us/ [Accessed 20 Dec 2019].

22 Ohkuma T, Fujii $\mathrm{H}$, Iwase $\mathrm{M}$, et al. Impact of sleep duration on obesity and the glycemic level in patients with type 2 diabetes: the Fukuoka Diabetes Registry. Diabetes Care 2013;36:611-7.

23 Ainsworth BE, Haskell WL, Whitt MC, et al. Compendium of physical activities: an update of activity codes and MET intensities. Med Sci Sports Exerc 2000;32:S498-516.

24 Ladloff L. The CES-D scale: a self-report depression scale for research in the general population. Appl Psychol Meas 1977;1:385-401. 
25 Kobayashi S, Murakami K, Sasaki S, et al. Comparison of relative validity of food group intakes estimated by comprehensive and brief-type self-administered diet history questionnaires against 16 d dietary records in Japanese adults. Public Health Nutr 2011;14:1200-11.

26 Matsuo S, Imai E, Horio M, et al. Revised equations for estimated GFR from serum creatinine in Japan. Am J Kidney Dis 2009;53:982-92.

27 Gan R-Y, Li H-B, Sui Z-Q, et al. Absorption, metabolism, anti-cancer effect and molecular targets of epigallocatechin gallate (EGCG): an updated review. Crit Rev Food Sci Nutr 2018;58:924-41.

28 Tajik N, Tajik M, Mack I, et al. The potential effects of chlorogenic acid, the main phenolic components in coffee, on health: a comprehensive review of the literature. Eur J Nutr 2017;56:2215-44.

29 Isogawa A, Noda M, Takahashi Y, et al. Coffee consumption and risk of type 2 diabetes mellitus. Lancet 2003;361:703-4.

30 Alperet DJ, Rebello SA, Khoo EY-H, et al. The effect of coffee consumption on insulin sensitivity and other biological risk factors for type 2 diabetes: a randomized placebo-controlled trial. Am J Clin Nutr 2020;111:448-58.

31 Greenberg JA, Boozer CN, Geliebter A, Coffee GA. Coffee, diabetes, and weight control. Am J Clin Nutr 2006;84:682-93.

32 Corti R, Binggeli C, Sudano I, et al. Coffee acutely increases sympathetic nerve activity and blood pressure independently of caffeine content: role of habitual versus nonhabitual drinking. Circulation 2002;106:2935-40.
33 Noordzij M, Uiterwaal CSPM, Arends LR, et al. Blood pressure response to chronic intake of coffee and caffeine: a meta-analysis of randomized controlled trials. J Hypertens 2005;23:921-8.

34 Kokubo Y, Iso $\mathrm{H}$, Saito I, et al. The impact of green tea and coffee consumption on the reduced risk of stroke incidence in Japanese population: the Japan public health center-based study cohort. Stroke 2013;44:1369-74.

35 Torres-Collado L, García-de la Hera M, Navarrete-Muñoz E, et al. Coffee drinking and associated factors in an elderly population in Spain. Int J Environ Res Public Health 2018;15:1661.

36 Strand BH, Grøholt E-K, Steingrímsdóttir OA, et al. Educational inequalities in mortality over four decades in Norway: prospective study of middle aged men and women followed for cause specific mortality, 1960-2000. BMJ 2010;340:c654.

37 Park S-Y, Freedman ND, Haiman CA, et al. Association of coffee consumption with total and cause-specific mortality among nonwhite populations. Ann Intern Med 2017;167:228-35.

38 Grosso G, Micek A, Godos J, et al. Coffee consumption and risk of all-cause, cardiovascular, and cancer mortality in smokers and non-smokers: a dose-response meta-analysis. Eur J Epidemiol 2016;31:1191-205.

39 Goto T, Nagashima H, Yoshida Y, et al. Contents of individual tea catechins and caffeine in Japanese green tea. Tea Res $J$ 1996;83:21-8. 